\title{
BIOLOGIA DE Aphis gossypii GLOVER, 1877 (Hemiptera: Aphididae) EM ABOBRINHA CULTIVAR CASERTA (Cucurbita pepo L.) EM DIFERENTES TEMPERATURAS ${ }^{1}$
}

\author{
Biology of Aphis gossypii Glover, 1877 (Hemiptera: Aphididae) on squash cultivar caserta \\ (Cucurbita pepo L.) in different temperatures
}

\author{
Melissa Vieira Leite ${ }^{2}$, Terezinha Monteiro dos Santos ${ }^{3}$, Brígida Souza 4 , \\ Ana Maria Calixto ${ }^{5}$, César Freire Carvalho ${ }^{4}$
}

\begin{abstract}
RESUMO
As cucurbitáceas apresentam grande demanda no mercado mundial e uma das principais pragas que atacam essas culturas é o pulgão Aphis gossypii. Assim, objetivou-se estudar aspectos da biologia desse afídeo em função da temperatura na cultivar de abobrinha 'Caserta' (Cucurbita pepo L.). Utilizaram-se discos foliares $(2,5 \mathrm{~cm}$ de $\varnothing$ ) acondicionados, com a face abaxial para cima, em placas de Petri $(5 \mathrm{~cm}$ de $\varnothing)$ contendo uma lâmina de ágar-água. Em cada placa foi deixada uma ninfa recém-nascida, acompanhandose o seu desenvolvimento ao longo de todo o ciclo de vida, em diferentes temperaturas $\left(18,21,24,27\right.$ e $\left.30 \pm 1^{\circ} \mathrm{C}\right), 70 \pm 10 \%$ UR e fotofase de 12 horas. O delineamento foi inteiramente casualizado, com 60 repetições. Verificou-se que as temperaturas afetaram o desenvolvimento de A. gossypii. Menor duração do período ninfal e maior produção diária de ninfas foram observadas a 24 e $27^{\circ} \mathrm{C}$. A temperatura de $30^{\circ} \mathrm{C}$ provocou efeito deletério, causando $68 \%$ de mortalidade na fase ninfal.
\end{abstract}

Termos para indexação: Pulgão, ciclo biológico, Cucurbitaceae.

\begin{abstract}
The cucurbitaceous plants are always on demand in world market and one of the main pests on these crop is the aphid Aphis gossypii. This work aimed to study the biological aspects of A. gossypii on the 'Caserta' (Cucurbita pepo L.) cultivar in different temperatures. To determine the biological aspects of $A$. gossypii, nymphs were kept on leaf disks $(2.5 \mathrm{~cm} \varnothing)$ with the abaxial side up in Petri dishes $(5 \mathrm{~cm} \varnothing)$ containing a thin layer of agar-water. Each dish contained one nymph of the first instar of the aphid. The development and the other biological parameters were evaluated in different temperatures $\left(18,21,24,27\right.$ e $\left.30 \pm 1^{\circ} \mathrm{C}\right), 70 \pm 10 \% \mathrm{RH}$ and $12 \mathrm{~h}$ photophase. The experiments were complete randomized design with 60 replicates. The results showed that the temperature influenced the development of $A$. gossypii. The shorter nymphal period and higher daily nymph's production were observed at 24 and $27^{\circ} \mathrm{C}$. The temperature of $30^{\circ} \mathrm{C}$ was deleterius inducing to $68 \%$ of mortality at the nymphal stage of $A$. gossypii.
\end{abstract}

Index terms: Aphid, biological cycle, Cucurbitaceae.

(Recebido em 26 de abril de 2006 e aprovado em 20 de novembro de 2006)

\section{INTRODUÇÃo}

O Brasil está inserido entre os quinze maiores produtores mundiais de algumas espécies de cucurbitáceas (Alvarenga \& Resende, 2002). Dados das Centrais de Abastecimento de Minas Gerais (CEASA/MG) revelaram uma oferta de $16.500 \mathrm{t}$ de abóbora no ano de 2005, com valor estimado de 6,3 milhões de reais, tendo $77 \%$ da produção sido das abobrinhas 'Caserta' e 'Menina Brasileira' (CEASA, 2005).
Entre as diversas pragas que ocasionam danos aos cultivos de cucurbitáceas destaca-se o pulgão Aphis gossypii Glover, 1877 (Hemiptera: Aphididae), considerado de grande importância pelos prejuízos que pode ocasionar (Cardoso, 1998; Gallo et al., 2002).

Os pulgões aglomeram-se, preferencialmente, na face inferior das folhas e nos brotos novos (Cardoso, 1998) e pela sucção de seiva ocasionam o encarquilhamento das folhas e deformação dos brotos, prejudicando o desenvolvimento da planta. Além disso, produzem o

\footnotetext{
${ }^{1}$ Projeto financiado pelo CNPq

${ }^{2}$ Doutoranda em Agronomia/Entomologia - Departamento de Entomologia/DEN - Universidade Federal de Lavras/UFLA - Cx. P. 3037 - $37200-000$ Lavras, MG - melissabio2000@yahoo.com.br

'Doutora em Entomologia Agrícola, Pesquisadora Agência Paulista de Tecnologia dos Agronegócios/APTA - Pólo Regional de Desenvolvimento Tecnológico dos Agronegócios do Extremo Oeste - Estrada Vicinal Nemezião de Souza Pereira, Km 06 - Cx. P. 67 - $16900-000$ - Andradina, SP Terezinha@aptaregional.sp.gov.br

${ }^{4}$ Doutor(a), Professor(a) - Departamento de Entomologia/DEN - Universidade Federal de Lavras/UFLA - Cx. P. 3037 - $37200-000$ - Lavras, MG brsouza@ufla.br; cfcarvalho@ufla.br

${ }^{5}$ Graduanda em Agronomia - Departamento de Entomologia/DEN - Universidade Federal de Lavras/UFLA - Cx. P. 3037 - $37200-000$ - Lavras, MG anamaria.calixto@bol.com.br
} 
honeydew, que propicia o desenvolvimento do fungo Capnodium spp., o que leva a formação de fumagina. Esta dificulta a respiração da planta e diminui a área fotossintética, contribuindo para o seu enfraquecimento. Contudo, as maiores perdas ocasionadas por esses insetos estão relacionadas à transmissão de vírus causadores de diversas enfermidades (Barbosa \& França, 1982).

A temperatura é um fator climático que afeta o desenvolvimento dos insetos e cada espécie apresenta uma exigência térmica que pode variar entre populações de regiões geográficas diferentes. Em geral, o tempo médio de desenvolvimento desses organismos decresce com o aumento da temperatura dentro da amplitude térmica requerida para a sobrevivência de cada espécie (Campbell \& Mackauer, 1975). Conforme Dixon (1987a), não apenas a velocidade de desenvolvimento, mas também a de reprodução é afetada pelos fatores extrínsecos relacionados à temperatura, bem como à qualidade do alimento.

Estudos realizados por Aldyhim \& Khalil (1993), Kocourek et al. (1994), Vansteenis \& El-Khawass (1995), Xia et al. (1999) e Soglia et al. (2003) evidenciaram que há variação dos aspectos biológicos de $A$. gossypii em função da temperatura do ambiente em que se desenvolvem. Assim, devido à importância do pulgão A. gossypii como praga das cucurbitáceas, bem como ao conhecimento relativamente restrito sobre sua biologia em plantas de abobrinha, o presente trabalho teve como objetivo estudar alguns aspectos biológicos desse afídeo na cultivar Caserta (Cucurbita pepo L.) em diferentes temperaturas.

\section{MATERIAL E MÉTODOS}

Para a realização dos experimentos foram empregadas plantas de abobrinha com cerca de 30 dias após a emergência. As folhas foram destacadas e lavadas em água corrente e posteriormente colocadas em solução de hipoclorito de sódio a $1 \%$ por cinco minutos para a desinfestação. Após este tempo, receberam duas lavagens em água destilada e, posteriormente, utilizando-se de um vazador metálico, confeccionaram-se discos foliares de 2,5 $\mathrm{cm}$ de diâmetro que foram acondicionados, com a face abaxial para cima, em placas de Petri de $5 \mathrm{~cm}$ de diâmetro contendo uma lâmina de aproximadamente $5 \mathrm{~mm}$ de agarágua a $1 \%$.

Transportou-se, para cada uma das 60 placas preparadas, uma fêmea adulta áptera de A. gossypii, proveniente de plantas de pepino (Cucumis sativus L.) As placas foram vedadas com filme de PVC laminado perfurado com estilete e, posteriomente, invertidas sobre uma bandeja para simular as condições naturais. Após 16 horas retirou-se cada fêmea e, das ninfas produzidas, uma foi selecionada aleatoriamente para a condução do experimento.

As placas de Petri contendo as ninfas de primeiro ínstar foram mantidas em câmaras climatizadas reguladas a $18,21,24,27$ e $30 \pm 1^{\circ} \mathrm{C}, 70 \pm 10 \%$ UR e fotofase de 12 horas. Os cinco tratamentos foram avaliados em 60 repetições em delineamento inteiramente casualizado.

Foram feitas avaliações a cada 24 horas observando-se a duração e viabilidade em cada estádio, duração do período ninfal, do ciclo biológico e viabilidade das ninfas. Na fase adulta avaliou-se a longevidade, a duração dos períodos pré-reprodutivo, reprodutivo, pósreprodutivo e a capacidade de reprodução, contando-se e retirando-se as ninfas diariamente.

Objetivando obter informações ao longo de todo o desenvolvimento de A. gossypii nas diferentes temperaturas, foram construídas curvas de sobrevivência, utilizando-se o estimador Kaplan-Meier não paramétrico (Colosimo, 2001). Foram comparadas as curvas de sobrevivência em cada ínstar e na fase adulta, considerando-se como eventos de interesse a mudança de ínstar das ninfas e morte dos adultos de A. gossypii. Assim, o tempo de permanência em cada ínstar até a ecdise da ninfa ou até a morte do adulto desse afídeo foram considerados como tempo de vida.

O tempo mediano de vida ( $\mathrm{T}$ ) é o tempo no qual pelo menos $50 \%$ dos indivíduos de uma amostra passaram pelo evento de interesse. Para a obtenção desse tempo, traçou-se uma reta paralela ao eixo x (tempo de vida), partindo de $0,5(50 \%)$ de sobrevivência, dada no eixo y (probabilidade de sobrevivência), até a interceptação com as curvas de sobrevivência. O ponto de encontro plotado no eixo x correspondeu ao tempo mediano. A porcentagem de sobrevivência $(\mathrm{S})$ correspondeu ao percentual de ninfas que não haviam passado para o ínstar ou fase subseqüente ou, no caso dos adultos, ainda não havia morrido, no tempo mediano.

Convencionou-se, neste estudo, utilizar a probabilidade de sobrevivência (S) verificada no tempo mediano e obtida traçando-se uma reta perpendicular ao eixo $\mathrm{x}$, a partir desse tempo, e plotando-se, no eixo y, o primeiro ponto de intersecção com as curvas de sobrevivência. Os valores exatos foram fornecidos pelo programa estatístico SAS (Proc Lifereg).

Os dados de duração da fase ninfal, longevidade, ciclo biológico, períodos reprodutivo e pós-reprodutivo, e número total de ninfas por fêmea, foram transformados em $\sqrt{x+1}$, em que $\mathrm{x}$ correspondeu à variável dependente. Com base nesses dados, foi feita a análise de variância (teste F) e as médias comparadas pelo teste de Tukey, a 5\% de 
significância. Os dados relativos à produção diária de ninfas/fêmea foram analisados por meio da mesma técnica, porém, sem transformação. Os dados obtidos para a viabilidade foram comparados utilizando-se intervalos de confiança de $95 \%$ para a diferença entre duas proporções.

\section{RESULTADOS E DISCUSSÃO}

No primeiro ínstar, as ninfas de A. gossypii apresentaram um tempo mediano de vida igual a dois, nas temperaturas de $18^{\circ} \mathrm{C}, 21^{\circ} \mathrm{C}$ e $24^{\circ} \mathrm{C}$. Porém, nas duas temperaturas mais baixas elas demoraram até três dias para mudarem de ínstar, o que não ocorreu a $24^{\circ} \mathrm{C}$. Nesta temperatura, todas as ninfas mudaram no segundo dia. A $27^{\circ} \mathrm{C}$, o tempo mediano de vida foi de um dia e, a $30^{\circ} \mathrm{C}$, de três dias. A maior duração deste ínstar foi de quatro dias, verificado para ninfas mantidas a $30^{\circ} \mathrm{C}$. As probabilidades de sobrevivência no tempo mediano de vida variaram de $0 \%$ a $48 \%$ para as diferentes temperaturas (Tabela 1, Figura 1A). Esses resultados evidenciam o efeito negativo da temperatura de $30^{\circ} \mathrm{C}$ sobre o desenvolvimento das ninfas, devido ao prolongamento deste estádio. Acrescenta-se, ainda, o fato de que $48 \%$ das ninfas ainda permaneciam neste ínstar no tempo mediano.

No segundo ínstar, o tempo mediano de vida foi de dois dias, para ninfas mantidas na menor temperatura, ou seja, o maior número de eventos ocorreu no segundo dia, com uma probabilidade de sobrevivência de $2 \%$. Houve um decréscimo do tempo mediano a $21^{\circ} \mathrm{C}, 24^{\circ} \mathrm{C}$ e $27^{\circ} \mathrm{C}$ e novo aumento a $30^{\circ} \mathrm{C}$ (Tabela 1 , Figura $1 \mathrm{~B}$ ). Observou-se uma tendência das temperaturas extremas afetarem a duração do segundo ínstar, ocasionando um prolongamento no desenvolvimento das ninfas criadas a $18^{\circ} \mathrm{C}$ e $30^{\circ} \mathrm{C}$, tendo a $30^{\circ} \mathrm{C}, 47 \%$ das ninfas ainda permanecido neste ínstar após o tempo mediano.
O tempo mediano de vida foi igual a dois dias a $18^{\circ} \mathrm{C}$ e $30^{\circ} \mathrm{C}$, no terceiro ínstar, e as probabilidades de sobrevivência de $0 \%$ e $30 \%$, respectivamente. Nas demais temperaturas, o tempo mediano foi de um dia, e as probabilidades de sobrevivência variaram de $12 \%$ a $45 \%$ (Tabela 1, Figura 1C). Constatou-se novamente o efeito negativo das temperaturas extremas, ocasionando uma maior duração em relação ao verificado nas demais condições. Contudo, a $18^{\circ} \mathrm{C}$, todas as ninfas mudaram de ínstar no segundo dia, e a $30^{\circ} \mathrm{C}, 30 \%$ delas ainda continuavam no terceiro estádio após o tempo mediano de vida, evidenciando um efeito negativo mais acentuado da temperatura de $30^{\circ} \mathrm{C}$ sobre as ninfas de $A$. gossypii.

As ninfas no quarto ínstar apresentaram tempo mediano de vida de dois dias a $18^{\circ} \mathrm{C}, 21^{\circ} \mathrm{C}$ e $30^{\circ} \mathrm{C}$, e de um dia a $24^{\circ} \mathrm{C}$ e $27^{\circ} \mathrm{C}$. As probabilidades de sobrevivência foram de $0 \%$ a $18^{\circ} \mathrm{C}$ e $21^{\circ} \mathrm{C}$, e de $20 \% ; 24 \%$ e $21 \%$ a $24^{\circ} \mathrm{C}, 27^{\circ} \mathrm{C}$ e $30^{\circ} \mathrm{C}$, respectivamente (Tabela 1, Figura 1D). Neste ínstar contatou-se um menor efeito das temperaturas extremas, em relação ao verificado nos ínstares anteriores. Contudo, a $30^{\circ} \mathrm{C}$, além de se verificar uma duração de dois dias para este ínstar, $21 \%$ das ninfas não atingiram a fase adulta no tempo mediano de vida.

De uma maneira geral, houve uma tendência de redução do tempo mediano de vida dos ínstares, com a elevação da temperatura na faixa de $18^{\circ} \mathrm{C}$ a $27^{\circ} \mathrm{C}$ e de um aumento deste tempo a $30^{\circ} \mathrm{C}$. Observou-se que a correlação negativa entre o período de desenvolvimento de $A$. gossypii e a temperatura ocorreu na faixa ótima de desenvolvimento desta espécie, que gira em torno de $27^{\circ} \mathrm{C}$, como demonstrado por Deguine (1995), citado por Fernandes et al. (2001).

A temperatura de $30^{\circ} \mathrm{C}$ exerceu um efeito negativo na biologia desse afídeo, provavelmente devido ao fato de que, conforme Campbell \& Mackauer (1975), altas

Tabela 1 - Tempo mediano de vida $\left(\mathrm{T}^{*}\right)$ (em dias) e probabilidade de sobrevivência $\left(\mathrm{S}^{* *}\right)$ (em \%) das ninfas e adultos de Aphis gossypii, em cinco temperaturas.

\begin{tabular}{|c|c|c|c|c|c|c|c|c|c|c|}
\hline \multirow{3}{*}{ Temperatura } & \multicolumn{8}{|c|}{ Ínstares } & \multirow{2}{*}{\multicolumn{2}{|c|}{ Adulto }} \\
\hline & \multicolumn{2}{|c|}{ Primeiro } & \multicolumn{2}{|c|}{ Segundo } & \multicolumn{2}{|c|}{ Terceiro } & \multicolumn{2}{|c|}{ Quarto } & & \\
\hline & $\mathrm{T}$ & $\mathrm{S}$ & $\mathrm{T}$ & $\mathrm{S}$ & $\mathrm{T}$ & $S$ & $\mathrm{~T}$ & $\mathrm{~S}$ & $\mathrm{~T}$ & $\mathrm{~S}$ \\
\hline $18^{\circ} \mathrm{C}$ & 2 & 7 & 2 & 2 & 2 & 0 & 2 & 0 & 35 & 45 \\
\hline $21^{\circ} \mathrm{C}$ & 2 & 3 & 1 & 32 & 1 & 34 & 2 & 0 & 17 & 49 \\
\hline $24^{\circ} \mathrm{C}$ & 2 & 0 & 1 & 18 & 1 & 12 & 1 & 20 & 20,5 & 50 \\
\hline $27^{\circ} \mathrm{C}$ & 1 & 7 & 1 & 3 & 1 & 45 & 1 & 24 & 12 & 37 \\
\hline $30^{\circ} \mathrm{C}$ & 3 & 48 & 3 & 47 & 2 & 30 & 2 & 21 & 9 & 42 \\
\hline
\end{tabular}

*T= tempo no qual ocorreu mudança de ínstar de $50 \%$ das ninfas ou morte de $50 \%$ dos adultos.

** $\mathrm{S}=$ probabilidade das ninfas não terem mudado de ínstar ou dos adultos não terem morrido até o tempo mediano. 

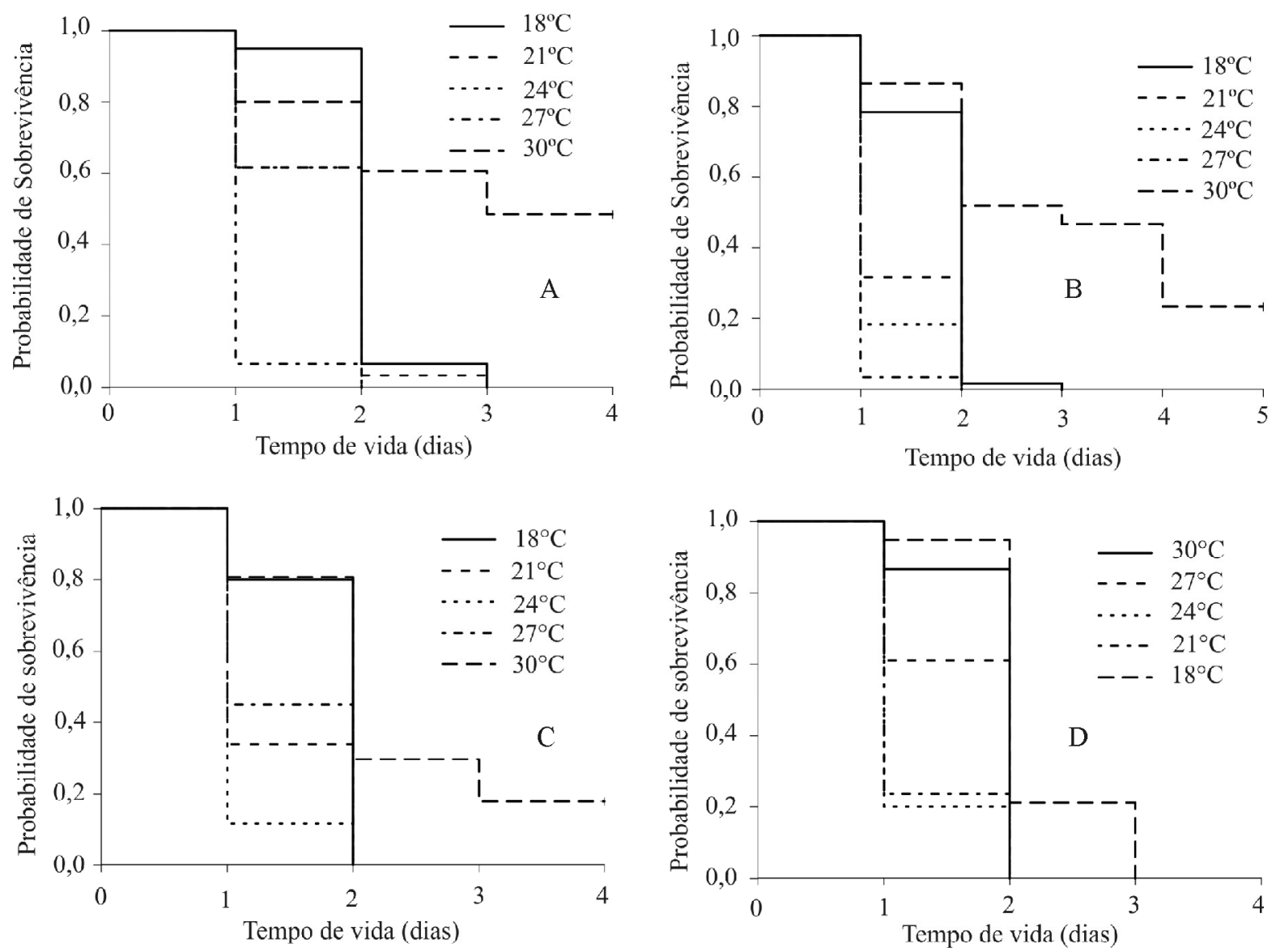

Figura 1 - Curvas de sobrevivência para o tempo mediano de vida (em dias) das ninfas de Aphis gossypii, em cinco temperaturas. A- primeiro ínstar; B- segundo ínstar; C- terceiro ínstar; D- quarto ínstar.

temperaturas podem resultar em morte por desnaturação de proteínas ou por distúrbios metabólicos causados pelo acúmulo de produtos tóxicos. Tais efeitos deletérios são verificados principalmente quando as altas temperaturas são mantidas constantes (Campbell et al., 1974). Na fase adulta, também observou-se um decréscimo do tempo mediano de vida com o aumento da temperatura verificandose uma maior longevidade a $18^{\circ} \mathrm{C}$ e menor a $30^{\circ} \mathrm{C}$. A $21^{\circ} \mathrm{C}$ houve uma exceção a esta tendência propiciando um tempo mediano de vida inferior ao observado a $24^{\circ} \mathrm{C}$ (Tabela 1 , Figura 2).

O pulgão A. gossypii apresentou quatro ínstares em todas as temperaturas, inclusive a $30^{\circ} \mathrm{C}$, fato que está de acordo com Dixon (1987b) quando afirmou que esta é uma característica biológica da maioria dos afídeos. Contudo, nessa temperatura, a maioria das ninfas morreu ao longo do desenvolvimento. Das 60 ninfas inicialmente individualizadas apenas 19 (31,7\%) atingiram a fase adulta, constatando-se maior mortalidade no segundo ínstar. Além disso, apenas seis dos 19 adultos produziram ninfas. Esse fato ocasionou uma heterogeneidade de variâncias na comparação das médias, optando-se, para fins de análise, por excluir os dados obtidos nesta temperatura.

Houve influência significativa das demais temperaturas sobre a fase ninfal desse afídeo, constatandose uma duração inversamente proporcional ao aumento da temperatura (Tabela 2). Este resultado assemelha-se aos obtidos por Aldyhim \& Khalil (1993) para A. gossypii criado em abóbora, por Kocourek et al. (1994) e Vansteenis \& ElKhawass (1995) para esse afídeo criado em pepino, por Kersting et al. (1999) e Xia et al. (1999) quando utilizaram algodoeiro como hospedeiro, e também por Soglia et al. (2002) que criaram esse pulgão em crisântemo.

$\mathrm{Na}$ fase adulta também se observou tendência de decréscimo da duração do período de vida com o aumento da temperatura. Houve diferença significativa entre as 


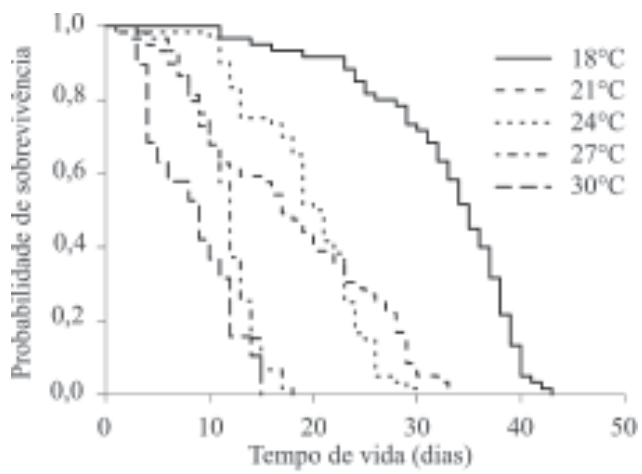

Figura 2 - Curvas de sobrevivência para o tempo mediano de vida (em dias) dos adultos de Aphis gossypii, em cinco temperaturas.

longevidades em função desse fator. No entanto, a $24^{\circ} \mathrm{C}$, a duração desse período foi maior que a $21^{\circ} \mathrm{C}$ (Tabela 2). Fato semelhante foi observado por Kersting et al. (1999), que constataram uma longevidade de 23,5 dias a $25^{\circ} \mathrm{C}$, e de 19,5 dias a $20^{\circ} \mathrm{C}$, para $A$. gossypii criado em algodoeiro.

A $18^{\circ} \mathrm{C}$, a duração da fase adulta de $A$. gossypii foi maior, correspondendo a aproximadamente o dobro do período de vida constatado a $21^{\circ} \mathrm{C}$. Esses resultados demonstram que temperaturas inferiores a $21^{\circ} \mathrm{C}$ causam acréscimos significativos na longevidade desse afídeo. Por outro lado, a elevação da temperatura a partir desse limiar térmico, embora geralmente seja seguida de reduções na longevidade, estas não são tão expressivas.

A duração do ciclo biológico também diminuiu com o aumento da temperatura constatando-se redução superior a 50\% no ciclo, com a elevação da temperatura de $18^{\circ} \mathrm{C}$ para $27^{\circ} \mathrm{C}$. No entanto, o ciclo biológico não foi afetado pelas temperaturas intermediárias, de $21^{\circ} \mathrm{C} \mathrm{e} 24^{\circ} \mathrm{C}$, evidenciando, mais uma vez, a menor sensibilidade dessa espécie de pulgão a variações dentro da faixa ótima de temperatura para o seu desenvolvimento (Tabela 2).

Para todas as temperaturas estudadas, a duração média do período pré-reprodutivo de $A$. gossypii foi inferior a 24 horas, não sendo possível verificar o efeito desse fator sobre esse parâmetro, haja vista que a primeira avaliação se procedeu após 24 horas na fase adulta.

O período reprodutivo tendeu a diminuir com o aumento da temperatura, exceto a $21^{\circ} \mathrm{C}$, condição que proporcionou a menor duração desse período (Tabela 3). Estes resultados aproximam-se dos de Vansteenis \& ElKhawass (1995) que obtiveram durações de 11,7; 11,5 e 10,4 dias para o período reprodutivo de A. gossypii criado em pepino a $20^{\circ} \mathrm{C}, 25^{\circ} \mathrm{C}$ e $30^{\circ} \mathrm{C}$, respectivamente. Soglia et al. (2003) também verificaram diminuição do período reprodutivo de A. gossypii criado em diferentes cultivares de crisântemo em função da temperatura, constatando-se redução próxima de 33\% com a elevação da temperatura de $25^{\circ} \mathrm{C}$ para $30^{\circ} \mathrm{C}$.

O período pós-reprodutivo também decresceu com o aumento da temperatura, sendo que a $18^{\circ} \mathrm{C}$ foi cerca de $60 \%$ superior ao constatado a $21^{\circ} \mathrm{C}$ e $24^{\circ} \mathrm{C}$, e $97,8 \%$ superior ao observado a $30^{\circ} \mathrm{C}$. Aldyhim \& Khalil (1993), trabalhando com abobrinha cultivar Clarita, verificaram períodos pósreprodutivos de 2,8; 1,7; 2,6; 2,3 e 5,9 dias a 10, 15, 20, 25 e $30^{\circ} \mathrm{C}$, respectivamente. Vansteenis \& El-Khawass (1995) também observaram uma duração de 2,5 dias a $20^{\circ} \mathrm{C}$ e $25^{\circ} \mathrm{C}$ e de 3,4 dias a $30^{\circ} \mathrm{C}$, em plantas de pepino.

Tabela 2 - Duração (em dias) (média \pm EP) da fase ninfal, longevidade e ciclo biológico de Aphis gossypii agora em quatro temperaturas.

\begin{tabular}{cccc}
\hline \multirow{2}{*}{ Temperatura } & \multicolumn{3}{c}{ Duração* } \\
\cline { 2 - 4 } & Fase ninfal & Fase adulta & Ciclo biológico \\
\hline $18^{\circ} \mathrm{C}$ & $7,47 \pm 0,07 \mathrm{a}$ & $32,16 \pm 0,98 \mathrm{a}$ & $39,76 \pm 0,98 \mathrm{a}$ \\
$21^{\circ} \mathrm{C}$ & $5,87 \pm 0,05 \mathrm{~b}$ & $16,36 \pm 1,17 \mathrm{c}$ & $22,63 \pm 1,17 \mathrm{~b}$ \\
$24^{\circ} \mathrm{C}$ & $5,11 \pm 0,05 \mathrm{c}$ & $18,90 \pm 0,75 \mathrm{~b}$ & $24,17 \pm 0,74 \mathrm{~b}$ \\
$27^{\circ} \mathrm{C}$ & $4,73 \pm 0,07 \mathrm{~d}$ & $11,19 \pm 0,42 \mathrm{~d}$ & $16,02 \pm 0,40 \mathrm{c}$ \\
\hline $\mathrm{P}$ & $<0,0001$ & $<0,0001$ & $>0,0001$ \\
\hline $\mathrm{CV}(\%)$ & 3,50 & 17,88 & 13,23 \\
\hline
\end{tabular}

*Médias seguidas pela mesma letra minúscula nas colunas não diferem significativamente entre si pelo teste de Tukey (P>0,05). $\mathrm{EP}=$ erro padrão

$\mathrm{P}=$ probabilidade de significância

$\mathrm{CV}=$ coeficiente de variação

Ciênc. agrotec., Lavras, v. 32, n. 5, p. 1394-1401, set./out., 2008 
Tabela 3 - Períodos reprodutivo e pós-reprodutivo (em dias) e produção total e diária (Média \pm EP) de ninfas por fêmea de Aphis gossypii, em quatro temperaturas.

\begin{tabular}{ccccc}
\hline \multirow{2}{*}{ Temperatura } & \multicolumn{2}{c}{ Período* } & \multicolumn{2}{c}{ Produção de ninfas* } \\
\cline { 2 - 5 } & Reprodutivo & Pós-reprodutivo & Total & Diária \\
\hline $18^{\circ} \mathrm{C}$ & $16,53 \pm 0,45 \mathrm{a}$ & $14,28 \pm 0,94 \mathrm{a}$ & $71,53 \pm 1,94 \mathrm{a}$ & $2,35 \pm 0,10 \mathrm{~d}$ \\
$21^{\circ} \mathrm{C}$ & $9,68 \pm 0,37 \mathrm{~d}$ & $5,74 \pm 0,95 \mathrm{~b}$ & $53,00 \pm 2,12 \mathrm{c}$ & $3,67 \pm 0,18 \mathrm{c}$ \\
$24^{\circ} \mathrm{C}$ & $12,81 \pm 0,32 \mathrm{~b}$ & $5,27 \pm 0,59 \mathrm{bc}$ & $73,66 \pm 1,90 \mathrm{a}$ & $4,09 \pm 0,14 \mathrm{~b}$ \\
$27^{\circ} \mathrm{C}$ & $10,38 \pm 0,33 \mathrm{bc}$ & $0,60 \pm 0,18 \mathrm{~d}$ & $55,45 \pm 2,19 \mathrm{~b}$ & $5,09 \pm 0,15 \mathrm{a}$ \\
\hline $\mathrm{P}$ & $<0,001$ & $<0,001$ & $<0,001$ & $<0,001$ \\
\hline $\mathrm{CV}$ & 11,61 & 39,25 & 14,37 & 30,41 \\
\hline
\end{tabular}

*Médias seguidas pela mesma letra minúscula nas colunas não diferem significativamente entre si pelo teste de Tukey $(\mathrm{P}>0,05)$.

$\mathrm{EP}=$ erro padrão

$\mathrm{P}=$ probabilidade de significância

$\mathrm{CV}=$ coeficiente de variação

O total de ninfas produzidas por fêmea foi maior a $18^{\circ} \mathrm{C}$ e a $24^{\circ} \mathrm{C}$. A menor produção foi observada a $21^{\circ} \mathrm{C}$, com uma média de 53 ninfas. Essa temperatura propiciou uma resposta não esperada para esse parâmetro avaliado, uma vez que ao longo do ciclo do pulgão, foi uma das condições mais propícias para o seu desenvolvimento. Diferentemente do constatado neste trabalho, os totais de ninfas obtidos por Vansteenis \& El-Khawass (1995), em plantas de pepino, aumentaram com a elevação da temperatura. Como relatado por Kocourek et al. (1994), o potencial reprodutivo dos afídeos pode ser afetado por vários fatores, salientando a qualidade da planta hospedeira, a ocorrência natural de resistência e a temperatura.

Por outro lado, o número de ninfas produzidas por dia foi diretamente proporcional ao aumento da temperatura. Essa correlação também foi verificada nos estudos de Vansteenis \& El-Khawass (1995) e Kersting et al. (1999). Xia et al. (1999) observaram esse mesmo comportamento na faixa de $10^{\circ} \mathrm{C}$ a $30^{\circ} \mathrm{C}$ mas, a $35^{\circ} \mathrm{C}$ houve diminuição na produção diária de ninfas, que assemelhouse à obtida a $10^{\circ} \mathrm{C}$. Soglia et al. (2003) observaram, nas três cultivares de crisântemo estudadas, maior fecundidade diária a $25^{\circ} \mathrm{C}$ e menor fecundidade nos extremos da faixa de temperatura avaliada $\left(15\right.$ e $\left.30^{\circ} \mathrm{C}\right)$.

Verificou-se que as temperaturas mais adequadas para o desenvolvimento de $A$. gossypii foram as de $24^{\circ} \mathrm{C}$ e $27^{\circ} \mathrm{C}$ uma vez que o desenvolvimento foi mais rápido e a produção diária de ninfas mais alta. A temperatura de $30^{\circ} \mathrm{C}$ teve um efeito deletério sobre o pulgão causando alta mortalidade na fase jovem, disfunções reprodutivas e alterações no formato dos insetos adultos caracterizadas pelo aspecto fusiforme apresentado por vários indivíduos. Estas constatações são embasadas nas afirmações de Dixon (1987a) que relatou que os afídeos possuem uma faixa ótima de temperatura em cujos extremos ocorre um aumento da mortalidade, desfavorecendo o desenvolvimento e a reprodução.

As ninfas de $A$. gossypii apresentaram praticamente $100 \%$ de viabilidade a $18,21,24$ e $27^{\circ} \mathrm{C}$, porém, a $30^{\circ} \mathrm{C}$, a viabilidade foi menor em todos os ínstares e, conseqüentemente, em toda fase ninfal, que apresentou viabilidade de $31,6 \%$ (Tabela 4). Ao contrário do constatado neste trabalho, Kersting et al. (1999) observaram $100 \%$ de viabilidade a $30^{\circ} \mathrm{C}$ e $0 \%$ a $35^{\circ} \mathrm{C}$, verificando-se maior tolerância dos indivíduos dessa população de pulgões em relação aos utilizados na presente pesquisa. Maior tolerância aos extremos de temperatura dentro da faixa de desenvolvimento, também foi constatada por Aldyhim \& Khalil (1993), que observaram uma viabilidade de $100 \%$ a 15,20 e $25^{\circ} \mathrm{C}$, de $80 \%$ a $30^{\circ} \mathrm{C}$ e de $0 \%$ a 10 e $35^{\circ} \mathrm{C}$. Esses resultados confirmam os efeitos deletérios de altas temperaturas que extrapolam a faixa ótima para o desenvolvimento da espécie. Por outro lado, Vansteenis \& El-Khawass (1995), testando as temperaturas 20, $25 \mathrm{e}$ $30^{\circ} \mathrm{C}$, e Soglia et al. (2002), utilizando $15,20,25$ e $30^{\circ} \mathrm{C}$ observaram que essas condições não influenciaram a viabilidade dos ínstares de A. gossypii criado em cultivares de pepino e de crisântemo, respectivamente. 
Tabela 4 - Viabilidade (\%) de cada ínstar das ninfas e da fase ninfal de Aphis gossypii, em cinco temperaturas.

\begin{tabular}{cccccc}
\hline \multirow{2}{*}{ Temperatura } & \multicolumn{4}{c}{ Viabilidade (\%) } \\
\cline { 2 - 5 } & $1^{\circ}$ ínstar & $2^{\circ}$ ínstar & $3^{\circ}$ ínstar & $4^{\text {o ínstar }}$ & Fase ninfal \\
\hline $18^{\circ} \mathrm{C}$ & $100 \mathrm{a}$ & $100 \mathrm{a}$ & $100 \mathrm{a}$ & $100 \mathrm{a}$ & $100 \mathrm{a}$ \\
$21^{\circ} \mathrm{C}$ & $100 \mathrm{a}$ & $100 \mathrm{a}$ & $98,3 \mathrm{a}$ & $100 \mathrm{a}$ & $98,3 \mathrm{a}$ \\
$24^{\circ} \mathrm{C}$ & $100 \mathrm{a}$ & $100 \mathrm{a}$ & $100 \mathrm{a}$ & $100 \mathrm{a}$ & $100 \mathrm{a}$ \\
$27^{\circ} \mathrm{C}$ & $100 \mathrm{a}$ & $100 \mathrm{a}$ & $100 \mathrm{a}$ & $98,3 \mathrm{a}$ & $98,3 \mathrm{a}$ \\
$30^{\circ} \mathrm{C}$ & $83,3 \mathrm{~b}$ & $74,0 \mathrm{~b}$ & $70,27 \mathrm{~b}$ & $73,0 \mathrm{~b}$ & $31,6 \mathrm{~b}$ \\
\hline
\end{tabular}

*Porcentagens seguidas pela mesma letra minúscula nas colunas não diferem significativamente entre si quando comparadas utilizando-se intervalos de $95 \%$ de confiança para proporção.

\section{CONCLUSÕES}

As temperaturas afetaram a biologia de A. gossypii criado em plantas de abobrinha cultivar Caserta, e as mais adequadas para o desenvolvimento desse afídeo foram as de $24^{\circ} \mathrm{C}$ e $27^{\circ} \mathrm{C}$ por propiciar redução do tempo de desenvolvimento e maior produção de ninfas. A temperatura de $30^{\circ} \mathrm{C}$ provocou efeito deletério sobre o pulgão, causando acentuada mortalidade na fase ninfal.

\section{REFERÊNCIAS BIBLIOGRÁFICAS}

ALDYHIM, Y. N.; KHALIL, A. F. Influence of temperature and daylength on population development of Aphis gossypii on Cucurbita pepo. Entomologia Experimentalis et Applicata, Dordrecht, v. 67, n. 2, p. 167-172, May 1993.

ALVARENGA, M. A. R.; REZENDE, G. M. A cultura do melão. Lavras: UFLA, 2002. 149 p.

BARBOSA, S.; FRANÇA, F. H. Pragas de cucurbitáceas e seu controle. Informe Agropecuário, Belo Horizonte, v. 8, n. 85 , p. 54-56, jan. 1982.

CAMPBELL, A.; FRAZER, B. D.; GILBERT, N.; GUITIERREZ, A. P.; MACKAUER, M. Temperature requeriments of some aphids and their parasites. Journal of Applied Ecology, Oxford, v. 11, n. 2, p. 431-438, 1974.

CAMPBELL, A.; MACKAUER, M. Thermal constants for development of the pea aphid (Homoptera: Aphididae) and same of its parasites. The Canadian Entomologist, Ottawa, v. 107, n. 4, p. 419-423, Apr. 1975.

CARDOSO, A. I. I. A cultura da abobrinha-de-moita. In: GOTO, R.; TIVELLI, S. W. Produção de hortaliças em ambientes protegidos: condições subtropicais, 1998. p. 105135.
CEASA. Disponível em:'<http://minas.ceasa.gov.bry. Acesso em: 15 nov. 2005

COLOSIMO, E. A. Análise de sobrevivência aplicada. In: REUNIÃO ANUAL DA REGIÃO BRASILEIRA DA SOCIEDADE INTERNACIONAL DE BIOMETRIA, 46; SIMPÓSIO DE ESTATISTICA APLICADA À EXPERIMENTAÇÃO AGRONÔMICA, 9., 2001, Piracicaba. Programas e resumos...Piracicaba: ESALQ/USP, 2001. 145 p.

DIXON, A. F. G. Parthenogenetic reproduction and rate of increase in aphids. In: MINKS, A. K.; HARREWINJN, P. World crop pest -Aphids: their biology, natural enemies and control. 1987a. v. 2A, Cap. 4. 5, p. 269-287.

DIXON, A. F. G. The way of life of aphids: host specificity, speciation and distribution. In: MINKS, A. K.; HARREWINJN, P. World crop pest-Aphids: Their biology, natural enemies and control. 1987b. v. 2A, Cap. 4. 5, p. 197-207.

FERNANDES, A. M. V.; FARIAS, A. M. I.; SOARES, M. M. M.; VASCONCELOS, S. D. Desenvolvimento do pulgão Aphis gossypii Glover (Hemiptera: Aphididae) em três cultivares de algodão herbáceo Gossypium hirsutum L. r. latifolium Hutch. Neotropical Entomology, Londrina, v. 30, n. 3, p. 467-470, July/Sept. 2001.

GALLO, D.; NAKANO, O.; SILVEIRA NETO, S.; CARVALHO, R. P. L.; BAPTISTA, G. C. de; BERTI FILHO, E.; PARRA, J. R. P.; ZUCCHI, R. A.; ALVES, S. B.; VENDRAMIM, J. D.; MARCHINI, L. C; LOPES, J. R. S.; OMOTO, C. Entomologia agrícola. São Paulo: Ed. FEALQ, 2002. v. 10.

KERSTING, U.; SATAR, S.; UYGUN, N. Effect of temperature on development rate and fecundity of apterous Aphis gossypii Glover (Hom., Aphididae) reared on Gossypium hirsutum L. Journal of Applied Entomology, Berlin, v. 123, n. 1, p. 23-27, Feb. 1999. 
KOCOUREK, F.; HAVELKA, J.; BERÁNKOVÁ, J.; JAROSIK, V. Effect of temperature on development rate and intrinsic rate of increase of Aphis gossypii reared in greenhouses cucumbers. Entomologia Experimentalis et Applicata, Dordrecht, v. 71, n. 1, p. 59-64, Apr. 1994.

SOGLIA, M. C. M.; BUENO, V. H. P.; RODRIGUES, S. M. M; SAMPAIO, M. V. Fecundidade e longevidade de Aphis gossypii Glover (Hemiptera, Aphididae) em diferentes temperaturas e cultivares comerciais de crisântemo. Revista Brasileira de Entomologia, Curitiba, v. 47, n. 1, p. 49-54, mar. 2003.

SOGLIA, M. C. M.; BUENO, V. H. P.; SAMPAIO, M. V. Desenvolvimento e sobrevivência de Aphis gossypii Glover
(Hemiptera: Aphididae) em diferentes temperaturas e cultivares comerciais de crisântemo. Neotropical Entomology, Londrina, v. 31, n. 2, p. 211-216, Apr./June 2002.

VANSTEENIS, M. J.; EL-KHAWASS, K. A. M. H. Life history of Aphis gossypii on cucumber: influence of temperature, host plant and parasitism. Entomologia Experimentalis et Applicata, Dordrecht, v. 76, n. 2, p. 121131, Aug. 1995.

XIA, J. Y.; WERF, W. van der; RABBINGE, R. Influence of temperature on bionomics of cotton aphid, Aphis gossypii, on cotton. Entomologia Experimentalis et Applicata, Dordrecht, v. 90, n. 1, p. 25-35, Jan. 1999. 